\title{
Unscented Kalman Filter for the Identification of Passive Control Devices
}

\author{
R. Ceravolo, A. De Stefano, E. Matta, A. Quattrone, L. Zanotti Fragonara \\ Department of Structural, Geotechnical and Building Engineering, Politecnico di Torino, 24 Corso Duca \\ degli Abruzzi, Torino, 10129, Italy
}

\begin{abstract}
The Unscented Kalman Filter (UKF) is a technique which allows dealing with nonlinear systems and it is able to handle any type of non-linearity. In detail, differently from Extended Kalman Filter $(\mathrm{EKF}), \mathrm{UKF}$ does not require the computation of the Jacobian of the non-linear function. Estimation of parameters through the UKF approach is an indirect procedure, consisting of transforming the parameter estimation problem into a state estimation problem. This is done by augmenting the system state vector by artificially defining the unknown parameters as additional state variables. In the present study the UKF is proposed to the purpose of the nonlinear identification of rolling-pendulum tuned vibration absorbers.
\end{abstract}

\section{INTRODUCTION}

In the field of nonlinear system identification several methods have been proposed in the past (Kerschen et al. 2006; Bursi et al. 2012). In the field of on-line methods the most promising are probably those belonging to the family of Kalman Filter algorithms. Though the Kalman Filter (1960) has been originally proposed for linear applications, different extensions of the method to the nonlinear field have been proposed.

The first group descends from the Extended Kalman Filter (EKF) (Jazwinski, 1966). The EKF allows applying the Kalman Filter to nonlinear optimal filtering. It forms a Gaussian approximation to the joint distribution of state and measurements by resorting to a Taylor series based transformation. When working with nonlinear processes the optimality of the estimation is lost and, even worst, the estimate may diverge (Stengel 1994). The EKF has some flaws: (i) it is not an optimal estimator; (ii) it may diverge due to its linearization approach if the initial estimate of the state vector is wrong or the process is modelled incorrectly; (iii) its estimated covariance matrix tends to underestimate the true covariance matrix so that it risks becoming inconsistent in the statistical sense without the addition of a "stabilising noise".

State-Dependent Riccati Equation Filter (SDRE) overcomes the EKF flaws, since it does not involve the Jacobian evaluations, but it entails direct parameterization (Beikzadeh \& Taghirad 2009). Briefly, it fully captures the nonlinearities of the system and brings the nonlinear system into a non- unique linear structure having state-dependent coefficients (SDCs). This nonuniqueness of the SDC form provides design flexibility which can be exploited to overcome serious difficulties such as singularities and loss of observability in traditional filtering methods.

Among the family of Particle Filters the most widely used and applied is the Unscented Kalman Filter (UKF). This technique has been recently proposed by Julier and Uhlman (2000) having the advantage, with respect to the canonical filters, of being able to treat any type of nonlinearity. In detail, UKF does not require the computation of the Jacobian of the nonlinear function; in fact it does not approximate the measurement equation of the system but it approximates the posterior probability density by a Gaussian density, by using a set of deterministic points (the so-called Sigma points). This allows dealing with also non differentiable nonlinear systems. When the Sigma points are propagated through the nonlinear transform, they capture the true mean and covariance of the Gaussian random variables, and the posterior mean and covariance accurately to the $3^{\text {rd }}$ order Taylor series expansion for any nonlinearity.

In the last few years, the UKF has been successfully applied for the identification of nonlinear mechanical systems in several numerical case studies, e.g. (Wu \& Smyth, 2008; Ceravolo et al. 2012). Due to the uncertainty in the dissipation laws of real dynamic systems (nonlinear damping, hysteresis, friction, etc.), very few experimental applications to real case studies are known. As a consequence, any ex- 
perimental applications should be previously validated through extensive numerical simulations.

The capability of UKF to deal with the nonlinear identification of system parameters has been tested on a numerical model aimed to describe the dynamic behaviour of a new prototype of vibro-protective control device recently proposed by Matta et al (2009). The device consists of an innovative type of rolling-pendulum tuned vibration absorber which, relying on an optimal three-dimensional guiding receptacle, can simultaneously control the response of the supporting structure along two orthogonal horizontal directions.

Through preliminary numerical simulations, the effectiveness of the UKF in identifying the structural parameters of the new device is investigated for different alternative descriptions of the nonlinear dissipation model introduced to simulate rolling friction at the interface between the ball bearing and the rolling surface.

\section{THE NEW BIDIRECTIONAL PENDULUM VIBRATION ABSORBER}

In recent years, many vibration control strategies have been developed for enhancing serviceability and safety of Civil Engineering structures against natural and manmade hazards. Among them, tuned vibration absorbers have been extensively studied and widely applied because of their simplicity, efficiency and low maintenance cost.

In its basic configuration, a tuned vibration absorber merely consists of a viscously damped singledegree-of-freedom (SDOF) auxiliary system attached to the main structure. By properly tuning its natural frequency to that of the selected target mode of the main structure, part of the vibratory energy of that mode is transferred to the auxiliary system and dissipated through its damping (Warburton \& Ayorinde, 1982).

Tuned absorbers have found through the years a variety of technological implementations (Matta \& De Stefano, 2009). Among them, the rocking pendulum, the ball pendulum and the rolling pendulum share the same idea of using curved rolling surfaces to derive the necessary restoring force, which makes them more compact and durable than hanging pendulums as well as more suitable to tuning to lowperiod structures for seismic applications. Several variants of rolling pendulum vibration absorbers are described in the literature (Legeza, 2005; Pirner, 2002), however arranged either in a planar configuration (constrained to move in a vertical plane) or in an axial-symmetrical configuration (constrained on a spherical cavity).

In order to simultaneously achieve bidirectional tuning to two distinct in-plan orthogonal structural modes, a new bidirectional pendulum vibration ab- sorber was proposed by the first author in (Matta et al. 2009), based on an innovative non-axialsymmetrical rolling guide. An analytical non-linear dynamic model was first derived using Appell's non-holonomic mechanics, and then a laboratory scaled prototype was tested which eventually demonstrated the bidirectional tuning capability and validated the mathematical model. Yet, the nonlinear dissipation law governing the rolling problem was outside the scope of the analytical model and therefore was only roughly identified from the experiments.

Aiming at more accurately characterizing the dissipative term, the same prototype absorber is here reconsidered. This consists of two identical plexiglass plates having dimensions $400 \times 300 \times 25 \mathrm{~mm}^{3}$, each carved with five identical toroidal concavities (Fig. 1). The plates are deployed with their concavities symmetrically facing each other, with three out of the five pairs of concavities sandwiching a stainless steel ball of radius $\mathrm{R}=10 \mathrm{~mm}$. The mass of each plate is $\mathrm{m}=3.42 \mathrm{~kg}$. The "physical" concavities have radii of curvature $R_{\mathrm{xp}}=29.9 \mathrm{~mm}$ and $\mathrm{R}_{\mathrm{yp}}=$ $54.7 \mathrm{~mm}$, and are $9 \mathrm{~mm}$ deep. Correspondingly, being $\mathrm{R}=10 \mathrm{~mm}$, the "virtual" radii are $\mathrm{R}_{\mathrm{x}}=19.9 \mathrm{~mm}$ and $\mathrm{R}_{\mathrm{y}}=44.7 \mathrm{~mm}$.
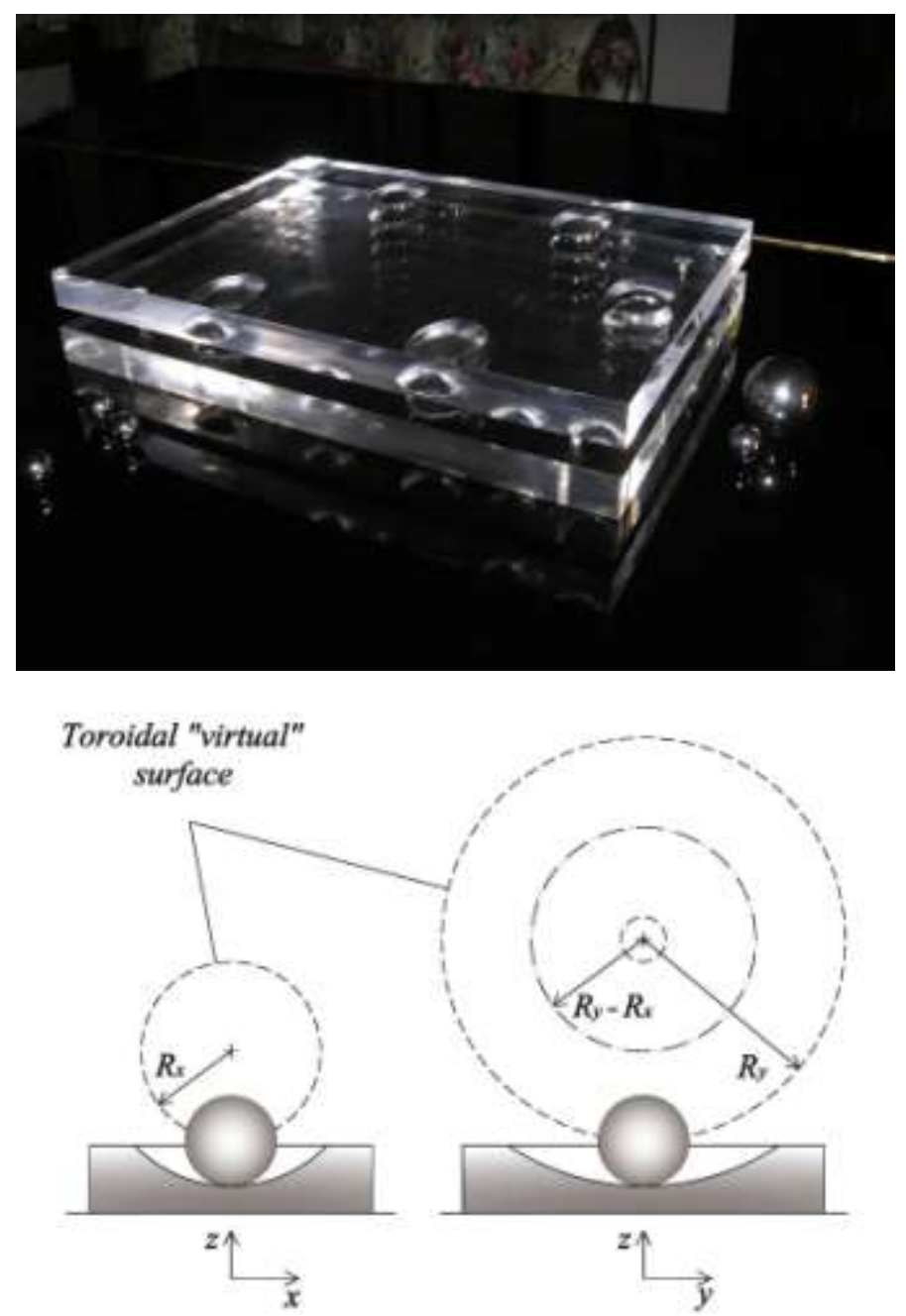

Figure 1. The prototype: (left) the test assembly; (right) a scheme of the toroidal concavities. 
In (Matta et al. 2009), with four accelerometers attached to each plate along the lateral faces (two in the $\mathrm{x}$ direction and two in the $\mathrm{y}$ direction), two kinds of experimental tests were conducted: fixed-base free motion tests, through imparting an initial nonzero relative displacement of the upper plate; and base shaking tests, through imparting a (nonstationary) random bidirectional translational motion to the lower plate. In the present paper, as an intermediate step towards using the UKF for the experimental identification of the dissipative rolling mechanism from those tests, the latter are preliminary replaced with numerical simulations, for simplicity circumscribed to unidirectional motions in the $y z$ vertical plane only.

\section{THE NONLINEAR MODEL}

The thorough nonlinear model describing the threedimensional rolling-without-slipping motion of each ball with respect to both the lower and the upper plates, and therefore of the upper plate with respect to the lower plate, is given in (Matta et al. 2009). As long as ball bearings' inertia is neglected and motion is restrained to the yz plane, that model reduces to the following nonlinear SDOF one (Matta \& De Stefano, 2009):

$\ddot{\theta}+c_{1}|\dot{\theta}|^{c_{2}} \operatorname{sgn}(\dot{\theta})+\frac{g}{R} \sin \theta=-\frac{1}{R} \ddot{x}_{g} \cos \theta$

where $\theta$ is the pendulum angular deflection, is the absolute acceleration of the lower plate, $R=2 R_{y}$ is the effective pendulum radius, $g$ is the gravity constant, $\mathrm{c}_{1}$ and $\mathrm{c}_{2}$ are the two parameters governing the dissipation mechanism, here assumed to be velocitydependent and someway intermediate between dry friction $\left(c_{2}=0\right)$ and linear viscous damping $\left(c_{2}=1\right)$, as suggested by experiments (Matta et al. 2009). Notice that, for the assigned value of $\mathrm{R}_{\mathrm{y}}$, the undamped linearized natural period, defined as $\mathrm{T}=2 \pi / \omega=$ $2 \pi \sqrt{ }(\mathrm{R} / \mathrm{g})$, happens to be exactly equal to $0.60 \mathrm{~s}$.

\section{UKF WITH BOUNDARIES}

The equations of a general dynamic system in discrete form can be defined by resorting to the statespace equation and its measurement equation as follows:

$$
\begin{aligned}
& \mathbf{X}_{k}=f\left(\mathbf{X}_{k-1}, u_{k-1}\right)+\mathbf{q}_{k} \\
& \mathbf{Y}_{k}=h\left(\mathbf{X}_{k}\right)+\mathbf{r}_{k}
\end{aligned}
$$

where $\mathbf{X}$ is the state vector in its augmented form, $\mathbf{Y}$ is the output vector, $u$ is the input whilst $\mathbf{q}$ and $\mathbf{r}$ are the process and measurement noises, respectively.

In this paper the UKF algorithm (see Table 1 or, for further information, (Julier et al. 2000)) is applied to the system described in Equation 1 using a modified version which allows for interval constraints on the state vector:

$$
\mathbf{X}_{L} \leq \mathbf{X}_{k} \leq \mathbf{X}_{U}
$$

Table 1. The UKF algorithm as proposed by Julier and Ulhmann (Julier et al. 2000)

1. Unscented transform and computation of sigma points

$$
\begin{gathered}
\mathscr{X}_{k-1}=\left[\begin{array}{ll}
\hat{\mathbf{X}}_{k-1} & \hat{\mathbf{X}}_{k-1}+\sqrt{(n+\lambda) \mathbf{P}_{X X_{k-1}}} \\
\ldots & \hat{\mathbf{X}}_{k-1}-\sqrt{(n+\lambda) \mathbf{P}_{X X_{k-1}}}
\end{array}\right]
\end{gathered}
$$

2. Nonlinear propagation of sigma points

$\mathscr{X}_{k, i}^{\mathscr{X}}=f\left(\mathscr{X}_{k-1}, u_{k-1}\right)$

3. State vector prediction

$\hat{\mathbf{X}}_{k}^{-}=\sum_{i=0}^{2 n} W_{i}^{(m)} \cdot \mathscr{X}_{k, i}^{\mathscr{X}}$

4. State covariance matrix prediction

$\mathbf{P}_{X X_{k}}^{-}=\sum_{i=0}^{2 n} W_{i}^{(c)}\left[\mathscr{X}_{k, i}^{\mathscr{X}}-\hat{\mathbf{X}}_{k}^{-}\right]\left[\mathscr{X}_{k, i}^{\mathscr{X}}-\hat{\mathbf{X}}_{k}^{-}\right]^{T}+\mathbf{Q}_{k}$

5. Propagation of sigma points through measure equation

$\mathscr{Y}_{k, i}=h\left(\mathscr{X}_{k, i}^{\mathcal{X}}\right)$

6. Measurement prediction

$\hat{\mathbf{Y}}_{k}^{-}=\sum_{i=0}^{2 n} W_{i}^{(m)} \cdot \mathscr{y}_{k, i}$

7. Measurement covariance matrix prediction

$\mathbf{P}_{Y Y_{k}}^{-}=\sum_{i=0}^{2 n} W_{i}^{(c)}\left[\mathscr{Y}_{k, i}-\hat{\mathbf{Y}}_{k}^{-}\right]\left[\mathscr{Y}_{k, i}-\hat{\mathbf{Y}}_{k}^{-}\right]^{T}+\mathbf{R}_{k}$

8. Cross covariance matrix prediction

$\mathbf{P}_{X Y_{k}}^{-}=\sum_{i=0}^{2 n} W_{i}^{(c)}\left[\mathscr{X}_{k, i}^{\mathscr{X}}-\hat{\mathbf{X}}_{k}^{-}\right]\left[\mathscr{Y}_{k, i}-\hat{\mathbf{Y}}_{k}^{-}\right]^{T}$

9. Kalman gain

$\mathbf{K}_{k}=\mathbf{P}_{x y}^{-}\left(\mathbf{P}_{y y}^{-}\right)^{-1}$

10. State vector update

$\hat{\mathbf{X}}_{k}=\hat{\mathbf{X}}_{k}^{-}+\mathbf{K}_{k}\left(\mathbf{Y}_{k}-\hat{\mathbf{Y}}_{k}^{-}\right)$

11. State covariance matrix update

$\mathbf{P}_{X X_{k}}=\mathbf{P}_{X X_{k}}^{-}-\mathbf{K}_{k} \mathbf{P}_{Y Y_{k}} \mathbf{K}_{k}^{t}$

In (Kolas et al. 2009) several steps were identified in the original UKF algorithm where constrains can be introduced: on the sigma points (step 1 and 2), on the state vector prediction (step3), on the output Sigma points (step 5), on the output (step 6) or on the updated state vector (step 10).

The bounds used in this paper have been applied according to the methods proposed in (Teixera et al. 2010) and in (Vachani et al. 2006). It has been cho- 
sen to deal with this problem by using both constraints on the unscented transform, on the predicted and updated state vector. In order to generate Sigma points satisfying Equation 3 one can use the following equations:

$$
\mathscr{X}_{k}=\mathbf{X}_{k}+\left[\begin{array}{ccc}
\mathbf{0}_{n x 1} & \theta_{1, k} \operatorname{col}_{1}\left[\left(\mathbf{P}_{X X_{k}}\right)^{1 / 2}\right] & \ldots \\
\ldots & \theta_{n, k} \operatorname{col}_{n}\left[\left(\mathbf{P}_{X X_{k}}\right)^{1 / 2}\right] & \ldots \\
\ldots & -\theta_{n+1, k} \operatorname{col}_{n+1}\left[\left(\mathbf{P}_{X X_{k}}\right)^{1 / 2}\right] & \ldots \\
\ldots & -\theta_{2 n, k} \operatorname{col}_{2 n}\left[\left(\mathbf{P}_{X X_{k}}\right)^{1 / 2}\right]
\end{array}\right]
$$

where $n$ is the length of the state vector and $\theta$ is defined as follows:

$$
\theta_{j, k}=\min \left(\operatorname{col}_{j}(\Theta)\right)
$$

$$
\Theta_{(i, j)}= \begin{cases}\sqrt{n+\lambda} & \text { if } \mathbf{S}_{(i, j)}=0 \\ \min \left(\sqrt{n+\lambda}, \frac{\mathbf{X}_{U, i}-\hat{\mathbf{X}}_{k}}{\mathbf{S}_{(i, j)}}\right) & \text { if } \mathbf{S}_{(i, j)}>0 \\ \min \left(\sqrt{n+\lambda}, \frac{\mathbf{X}_{L, i}-\hat{\mathbf{X}}_{k}}{\mathbf{S}_{(i, j)}}\right) & \text { if } \mathbf{S}_{(i, j)}<0\end{cases}
$$

$$
\mathbf{S}=\left[\begin{array}{ll}
\left(\mathbf{P}_{X X_{k}}\right)^{1 / 2} & -\left(\mathbf{P}_{X X_{k}}\right)^{1 / 2}
\end{array}\right]
$$

Weights applied to the Sigma points are given by $W_{i}=\left[\begin{array}{llll}W_{0, k} & W_{1, k} & \ldots & W_{2 n, k}\end{array}\right]$ satisfying

$\sum_{j=0}^{2 n} W_{1, k}=1$ where:

$W_{0, k}=b_{k}, \quad W_{j, k}=a_{k} \cdot \theta_{j, k}+b_{k}$

with $a_{k}$ and $b_{k}$ determined using the equation proposed by Teixera (2010). Furthermore, in order to apply the constraints on step 3 and 10, a simple clipping procedure on the vector $\mathbf{X}_{k}$ is performed. It is worth noticing that the clipping procedure, even if it is rough, is the optimal solution in most of the usual problems (see (Vachani et al. 2006)) and without doubt the most computationally efficient.

\section{NUMERICAL EXAMPLES}

The UKF is here applied to estimate the two states and the two dissipation coefficients $c_{1}$ and $c_{2}$ of the nonlinear model defined by Equation 1, i.e. to identify the following joint state vector:
$\mathbf{X}=\left[x_{1}, x_{2}, x_{3}, x_{4}\right]=\left[x, \dot{x}, c_{1}, c_{2}\right]$

while the two remaining parameters, $g$ and $R$, are assumed to be exactly known. For the sake of validation, the identification is repeated for a number of simulated scenarios, chosen to roughly reproduce the two experimental test cases, i.e. the free decay oscillation and the random base shaking. Namely, three distinct models are simulated, each described by a different pair $\left[c_{1}, c_{2}\right]$ of the model parameters, and each responding to the experimental inputs in nearly the same way as the real device, as can be appreciated from Figure 2. For each model, the UKF identification is then repeatedly run for different additive measurement noise levels and different initial estimation errors.
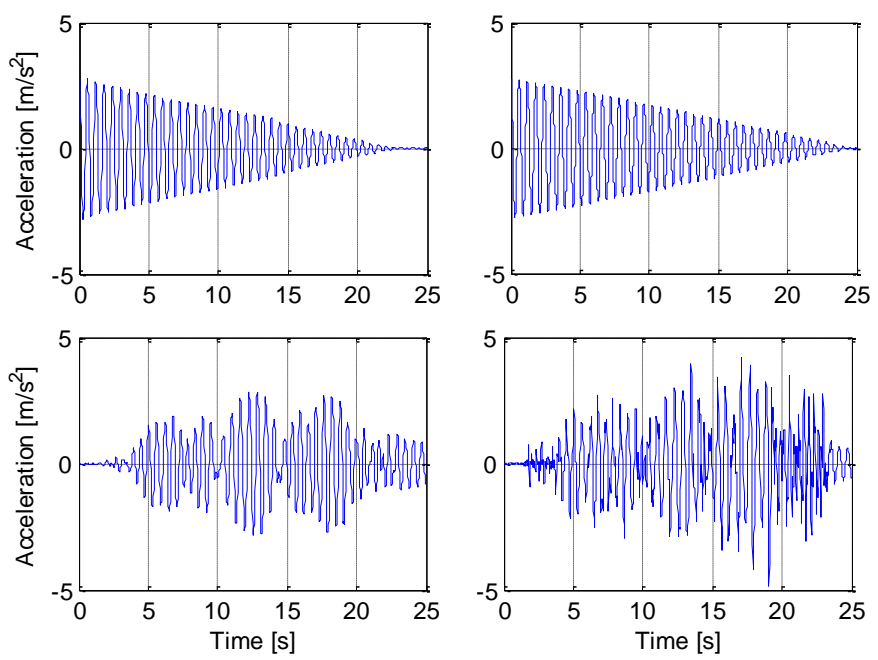

(a)

(b)
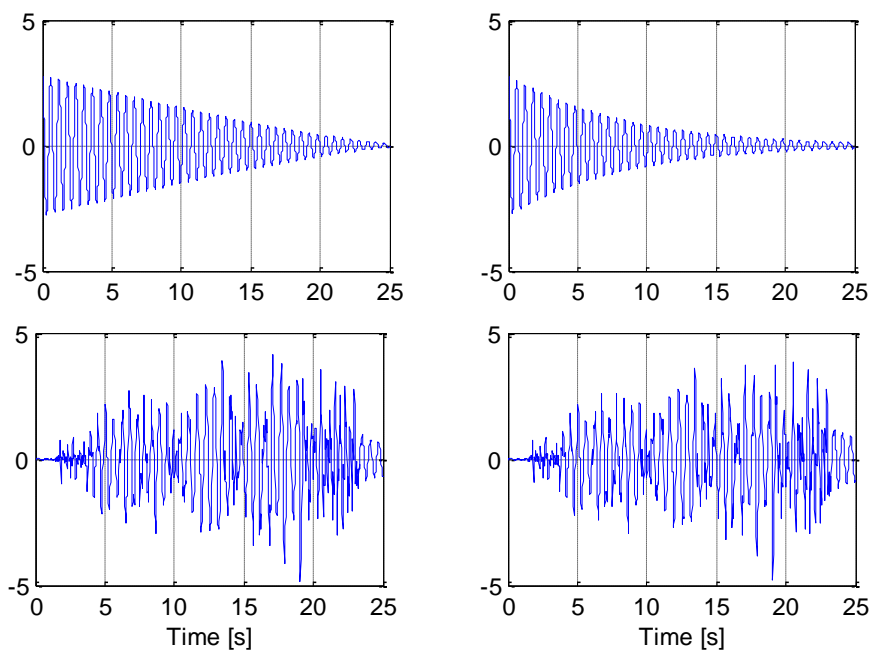

(c)

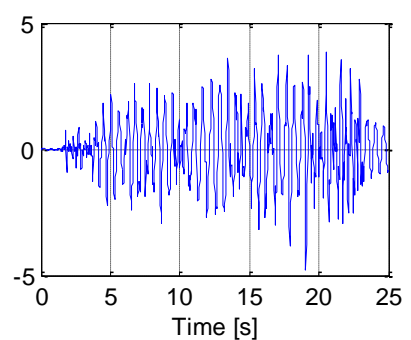

(d)

Figure 2. The free decay (above) and random shaking (bottom) responses of: (a) the real test device; (b) the model with $c_{1}=$ 0.2 and $c_{2}=0.0$; (c) the model with $c_{1}=0.2$ and $c_{2}=0.3$; (d) the model with $c_{1}=0.25$ and $c_{2}=1.0$.

Some of the results obtained for the free decay input scenario are exemplified in Figure 3. 


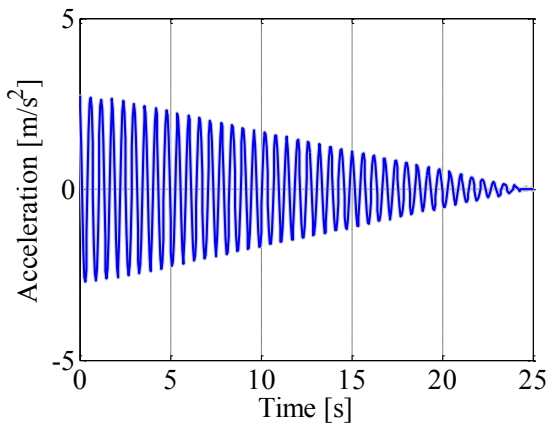

(a)

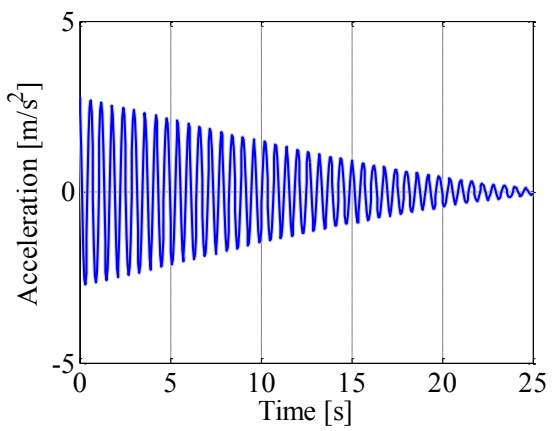

(b)

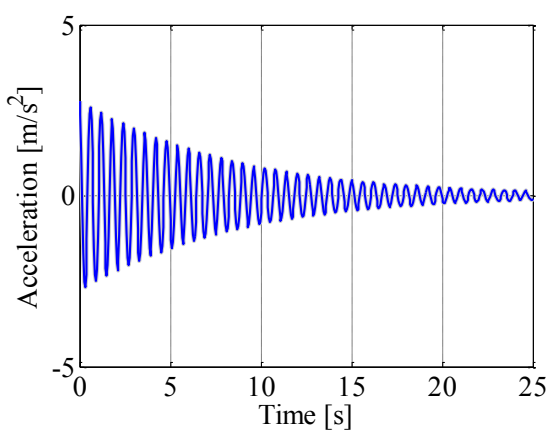

(c)

Figure 3. UKF matching of the free decay response for: (a) $c_{1}=$ 0.2 and $c_{2}=0.0$; (b) $c_{1}=0.2$ and $c_{2}=0.3$; (c) $c_{1}=0.25$ and $c_{2}=$ 1.0 - Simulated model (thick grey line) vs. estimated model (thin blue line).
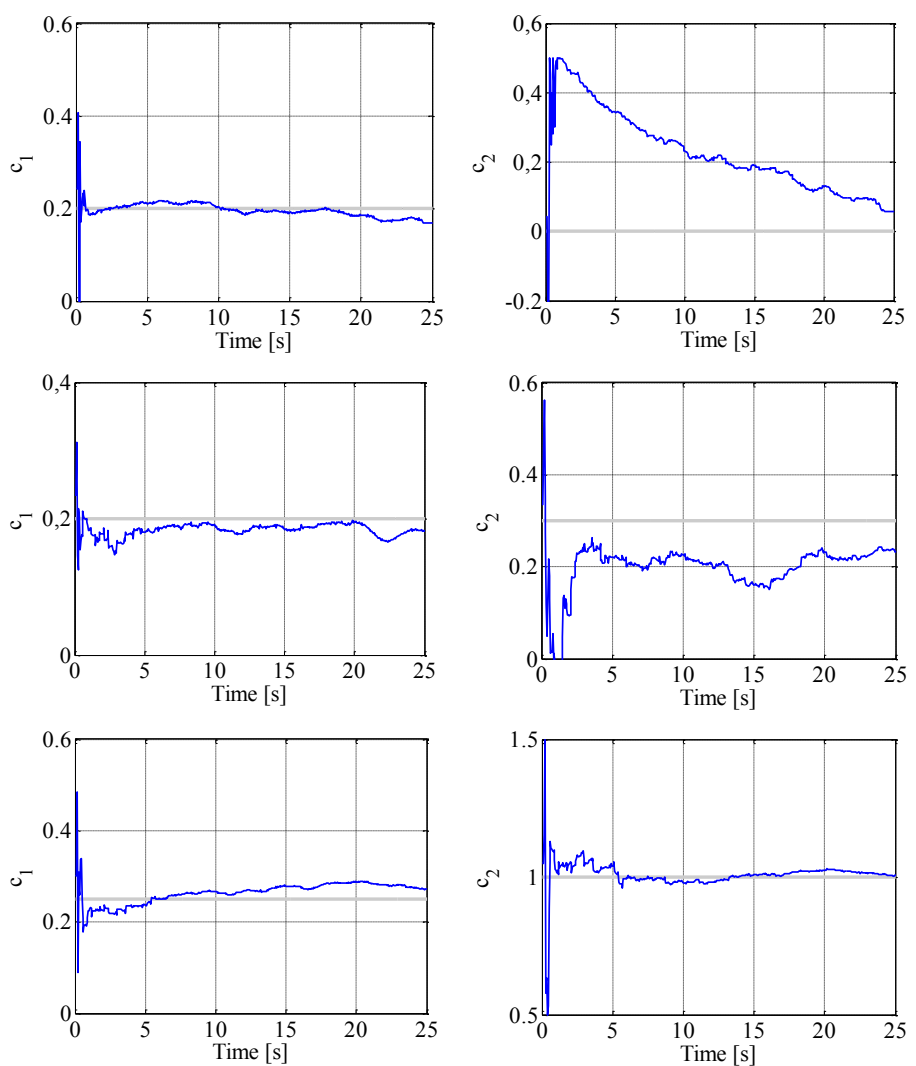

Figure 4. UKF esteems from the free decay response for: (above) $\mathrm{c} 1=0.2$ and $\mathrm{c} 2=0.0 ;$ (middle) $\mathrm{c} 1=0.2$ and $\mathrm{c} 2=0.3$; (bottom) $\mathrm{c} 1=0.25$ and $\mathrm{c} 2=1.0-$ Simulated model (thick grey line) vs. estimated model (thin blue line).

One example of the results obtained for the random shaking input scenario is reported in Figure 4.

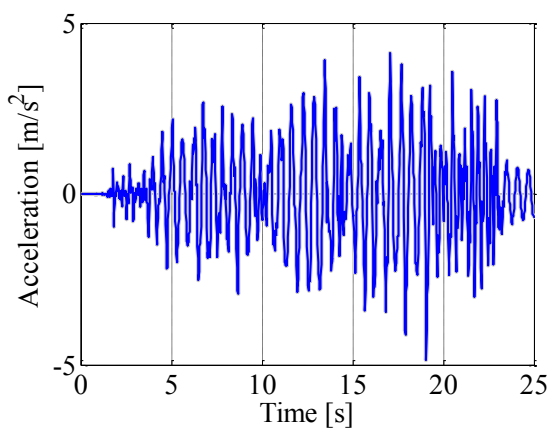

(a)

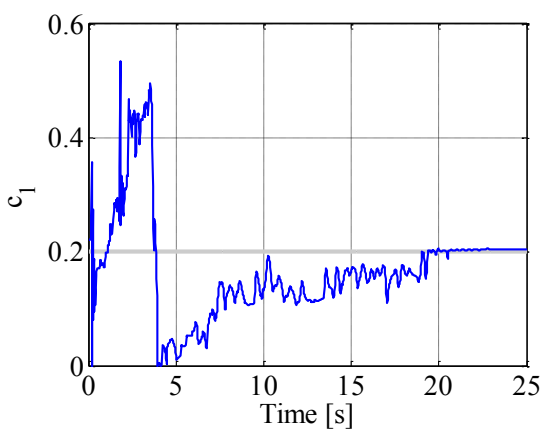

(b)

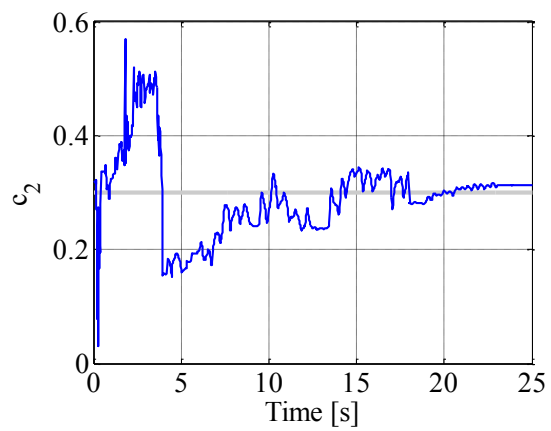

(c)

Figure 5. UKF applied to the random shaking response for $c_{1}=$ 0.2 and $c_{2}=0.3$ - Simulated model (thick grey line) vs. estimated model (thin blue line).

For both the free decay and the random shaking scenarios, a satisfactory performance of the UKF estimation can be generally appreciated. The simulated response can be tracked with good accuracy and the estimated parameters tend to rapidly converge to the assumed numerical values in nearly all cases. This is a promising result in view of the application to the real experimental data, which is left for future work.

\section{CONCLUSIONS}

In the present work, a preliminary numerical investigation is reported in order to assess the viability of UKF for the nonlinear identification of a new prototype of vibro-protective control device. The device consists of an innovative type of rolling-pendulum tuned vibration absorber which, relying on an optimal three-dimensional guiding receptacle, can si- 
multaneously control the response of the supporting structure along two orthogonal horizontal directions.

Through preliminary numerical simulations, the effectiveness of the UKF in identifying the structural parameters of the new device is investigated for different alternative descriptions of the nonlinear dissipation model introduced to simulate rolling friction at the interface between the ball bearing and the rolling surface. The experimental characterization of the real test prototype is left for future work.

\section{REFERENCES}

Beikzadeh, H. and Taghirad, H.D. 2009. Robust $\mathrm{H} \infty$ Filtering for Nonlinear Uncertain Systems using State-Dependent Riccati Equation Technique. Joint 48th IEEE Conference on Decision and Control and 28th Chinese Control Conference.

Bursi, O.S., Ceravolo, R., Erlicher, S. and Zanotti Fragonara, L. 2012. Identification of the hysteretic behaviour of a partial-strength steel-concrete moment-resisting frame structure subject to pseudodynamic tests. Earthquake Engineering \& Structural Dynamics 41(14): 1883-1903.

Ceravolo, R., Matta, E., Quattrone, A., Surace, C. and Zanotti Fragonara, L. 2012. On-line identification of time-varying systems equipped with adaptive control. Journal of Physics: Conference Series 382.

Jazwinski, A.H. 1966. Filtering for nonlinear dynamical systems. IEEE Transactions on Automatic Control 11(4): 765-766.

Julier, S.J., Uhlmann, J.K. and Durrant-Whyte, H.F. 2000. A new method for the nonlinear transformation of means and covariances in filters and estimators. IEEE Trans. Automat. Control 45(3): 477-482.

Kalman, R.E. 1960. A New Approach to Linear Filtering and Prediction Problems. ASME Journal of Basic Engineering 82: 35-45.

Kerschen, G., Worden, K., Vakakis, A.F. and Golinval, J.C. 2006. Past, present and future of nonlinear system identification in structural dynamics. Mechanical Systems and Signal Processing 20: 505-592.

Kolas, S., Foss, B.A. and Schei, T.S. 2009. Constrained nonlinear state estimation based on the UKF approach. Computers and Chemical Engineering 33: 1386-1401.

Legeza, V.P. 2005. Analytic determination of the amplitude-frequency characteristic of a nonlinear vibroprotective system with roller damper. Strength of Materials 37(2): 214-224.

Matta, E. and De Stefano, A. 2009. Seismic performance of pendulum and transaltional roofgarden TMDs. Mechanical Systems and Signal Processing 23: 908-921.
Matta, E., De Stefano, A. and Spencer, B.F.J. 2009. A new passive rolling-pendulum vibration absorber using a non-axial-symmetrical guide to achieve bidirectional tuning. Earthquake Engineering \& Structural Dynamics 38: 1729-1750.

Pirner, M. 2002. Actual behaviour of a ball vibration absorber. Journal of Wind Engineering and Industrial Aerodynamics 90: 987-1005.

Stengel, R.F. 1994. Optimal Control and Estimation. Dover.

Teixera, B.O.S., Torres, L.A.B., Aguirre, L.A. and Bernstein, D.S. 2010. On unscented Kalman filtering with state interval constraint. Journal of Process Control 20: 45-57.

Vachani, P., Narasimhan, S. and Rengaswamy, R. 2006. Robust and reliable estimation via Unscented Recursive Nonlinear Dynamic Data Reconciliation. Journal of Process Control 16: 1075-1086.

Warburton, G.B. and Ayorinde, E.O. 1982. Optimum absorber parameters for various combination of response and excitation parameters. Earthquake Engineering \& Structural Dynamics 10: 381-401.

$\mathrm{Wu}, \mathrm{M}$. and Smyth, A.W. 2008. Real-time parameter estimation for degrading and pinching hysteretic models. International Journal of NonLinear Mechanics 43: 822-833. 
2013-12-31

\section{Unscented kalman filter for the} identification of passive control devices

Ceravolo, Rosario

Taylor \& Francis

Rosario Ceravolo, Alessandro De Stefano, Emiliano Matta, Antonino Quattrone and Luca

Zanotti Fragonara. Unscented kalman filter for the identification of passive control devices.

Proceedings of the Fifth International Conference on Structural Engineering and Computation, 2-4 September 2013, Cape Town, South Africa.

https://dspace.lib.cranfield.ac.uk/handle/1826/12255

Downloaded from Cranfield Library Services E-Repository 